\title{
Quantitative assessment of renal allograft pathologic changes: comparisons of mono-exponential and bi-exponential models using diffusion-weighted imaging
}

\author{
Min Fan ${ }^{1 \#}$, Zhaoyu Xing ${ }^{1 \#}$, Yanan $\mathrm{Du}^{2}$, Liang $\operatorname{Pan}^{2}$, Yangyang Sun ${ }^{1}$, Xiaozhou He ${ }^{1}$ \\ ${ }^{1}$ Department of Urology, ${ }^{2}$ Department of Radiology, the Third Affiliated Hospital of Soochow University, Changzhou 213003, China \\ \#These authors contributed equally to this work.
}

Correspondence to: Xiaozhou He; Yangyang Sun. Department of Urology, the Third Affiliated Hospital of Soochow University, 185 Juqian Street, Changzhou 213003, China. Email: fnmong@hotmail.com; Syy90614@126.com.

Background: Diffusion-weighted imaging (DWI) can noninvasively assess renal allograft pathologic changes that provide useful information for clinical management and prognostication. However, it is still unknown whether the bi-exponential model analysis of DWI signals is superior to that of the monoexponential model.

Methods: Pathologic and DWI data from a total of 47 allografts were prospectively collected and analyzed. Kidney transplant interstitial fibrosis was quantified digitally. The severity of acute and chronic pathologic changes was semi-quantified by calculating the acute composite scores (ACS) and chronic composite score (CCS). Mono-exponential total apparent diffusion coefficient (ADCT), and the bi-exponential parameters of true diffusion (D) and perfusion fraction (fp) were acquired. The diagnostic performances of both monoexponential and bi-exponential parameters were assessed and compared by calculating the area under the curve (AUC) from receiver-operating characteristic (ROC) curve analysis.

Results: ADCT, D, and fp were all significantly correlated with interstitial fibrosis, ACS, and CCS. Cortical fp discriminated mild from moderate and severe ACS with the largest AUC of 0.89 [95\% confidence interval (CI), 0.77-0.96]. Noticeably, only cortical fp could differentiate severe ACS from mild-to-moderate ACS $(\mathrm{P}<0.001)$ with an AUC of 0.80 (95\% CI, 0.65-0.90) and a sensitivity of $100 \%$ (95\% CI, 66.4-100\%). Strikingly, the joint use of D and fp in either the cortex or the medulla could achieve a sensitivity of $100 \%$ for identifying either mild or severe interstitial fibrosis. Meanwhile, the serial use of cortical D and cortical fp showed the largest specificity for identifying both mild [88.9\% (95\% CI, 70.8-97.6\%)] and severe [84.4\% (95\% CI, 67.2-94.7\%)] interstitial fibrosis. For identifying mild CCS, the AUC of medullary ADCT (0.90, 95\% CI, 0.78-0.97) was similar to that of cortical D (0.81, 95\% CI, 0.67-0.91) and fp (0.86, 95\% CI, 0.730.94), but statistically larger than that of medullary $\mathrm{D}(\mathrm{P}=0.005)$ and $\mathrm{fp}(\mathrm{P}=0.01)$. Furthermore, the parallel use of cortical D and cortical fp could increase the sensitivity to $95.0 \%$ (95\% CI, 75.1-99.9\%), whereas serial use of medullary D and medullary fp could increase the specificity to $100 \%$ (95\% CI, 87.2-100\%). The AUCs for differentiating severe from mild and moderate CCS were statistically insignificant among all parameters in the cortex and medulla $(\mathrm{P} \geq 0.15)$.

Conclusions: Cortical fp was superior to the ADCT for identifying both mild and severe acute pathologic changes. Nevertheless, ADCT was equal to or better than single D or fp for evaluating chronic pathologic changes. Thus, both monoexponential and bi-exponential analysis of DWI images are complementary for evaluating kidney allograft pathologic changes, and the combined use of $\mathrm{D}$ and fp can increase the sensitivity and specificity for discriminating allograft pathologic changes severity. 
Keywords: Diffusion-weighted imaging (DWI); kidney transplant; pathology

Submitted Nov 24, 2019. Accepted for publication May 11, 2020.

doi: $10.21037 /$ qims-19-985a

View this article at: http://dx.doi.org/10.21037/qims-19-985a

\section{Introduction}

With advances in perioperative management, the short-term survival rate of kidney transplants has improved dramatically. Counterintuitively, no significant improvements in longterm prognosis have been documented (1). Post-transplant complications, such as rejection and glomerulonephritis, could reduce the allograft survival rate to varying degrees. Both acute (e.g., inflammation) and chronic (e.g., interstitial fibrosis) pathologic changes are common histologic features in biopsy samples from dysfunctional allografts. These pathophysiologic processes reduce the viable number of nephrons and are potent predictors of allograft prognosis (2). Furthermore, the evaluation of allograft pathologic changes also bears therapeutic significance. For example, clinicians tend to treat patients with severe allograft interstitial fibrosis more conservatively, while more aggressive therapies would be much more likely to be offered to those with only mild tubulointerstitial fibrosis. Regrettably, the evaluation of kidney allograft pathologic changes could only be made by biopsies, which is an invasive procedure with potential complications of bleeding, infections, arteriovenous fistula formations, or even death. Therefore, the noninvasive quantification of kidney transplant pathologic changes would significantly facilitate patient management and prognostication assessment.

Diffusion-weighted imaging (DWI) is beneficial in the evaluation of kidney transplants, allowing a noninvasive means of kidney allograft functional characterization (3). Prior studies have consistently found that the apparent diffusion coefficient (ADC) calculated from DWI with a monoexponential model correlated significantly with interstitial fibrosis $(4,5)$. Nonetheless, monoexponential ADC does not consider the physiological process of microcapillary perfusion. By contrast, recent advances suggest that the bi-exponential models could more accurately characterize the molecular diffusion of water than mono-exponential models by separating true diffusion from pseudodiffusion caused by the blood microcirculation in capillaries. A recent study by Mao et al. (6) reported that intravoxel incoherent motion DWI (IVIM-DWI) could also characterize pathologic lesions in the kidneys. However, the capability of DWI parameters derived from both monoexponential and bi-exponential models to assess kidney allograft pathologic changes has not been compared, and it remains unclear which model is superior for categorizing kidney transplant pathologic changes. Moreover, earlier studies have focused on evaluating kidney fibrosis (4-8), but few have explored the capability of DWI to evaluate acute pathologic changes.

Thus, this study aimed to compare the diagnostic performances of bi-exponential IVIM-DWI with that of mono-exponential data analysis for evaluating the severity of pathologic changes in a cohort of kidney transplant patients, using histopathology as the reference standard.

\section{Methods}

\section{Patients}

This prospective study was approved by the local institutional review board, and written informed consent was obtained from all patients. Patients with allograft dysfunction (rising serum creatinine and/or proteinuria) who required allograft biopsy for differential diagnoses between July 2017 and April 2018 were recruited and examined. Patients with general contraindications to magnetic resonance imaging (MRI) were excluded from participation $(n=4)$. In total, 50 patients were examined, of which 3 cases were excluded from the final analysis due to a lack of biopsy $(n=1)$ and severe image motion artifacts $(n=2)$. Finally, data from 47 patients (33 males and 14 females, mean age $38.2 \pm 10.9$ years, and age range $18-63$ years) were analyzed. The demographics and baseline characteristics of the included patients are shown in Table 1.

\section{MRI acquisition}

All patients underwent MRI imaging using a 3.0 Tesla clinical MRI imaging unit (Discovery MR 750; General Electric Medical Systems, Milwaukee, WI, USA) and a 32-channel torso coil. Conventional coronal T1- and axial 
Table 1 Demographics and baseline characteristics of the included patients

\begin{tabular}{|c|c|}
\hline Characteristics & Values \\
\hline Age (years) & $38.2 \pm 10.9$ \\
\hline Male: female, n (\%) & 33 (70.2): 14 (29.8) \\
\hline \multicolumn{2}{|l|}{ Immunosuppressive regimen, n (\%) } \\
\hline Pre + MMF + FK & $28(59.6)$ \\
\hline Pre + MMF + CsA & $13(27.7)$ \\
\hline Other & $6(12.7)$ \\
\hline \multicolumn{2}{|l|}{ Causes of end-stage renal disease } \\
\hline Glomerulonephritides, n (\%) & $10(21.3)$ \\
\hline Vasculitis, n (\%) & $1(2.1)$ \\
\hline Unknown, n (\%) & $36(76.6)$ \\
\hline $\begin{array}{l}\text { Serum creatinine, median (interquartile } \\
\text { range) }\end{array}$ & $2.19(1.35-2.97)$ \\
\hline Proteinuria, median (interquartile range) & $0.67(0.33-2.38)$ \\
\hline Hemoglobin, g/L & $115.6 \pm 25.6$ \\
\hline $\begin{array}{l}\text { Interstitial fibrosis, median (interquartile } \\
\text { range) }\end{array}$ & $30.1 \%(8.9-58.3 \%)$ \\
\hline \multicolumn{2}{|l|}{ ACS, n (\%) } \\
\hline Mild ACS & $24(51.1)$ \\
\hline Moderate ACS & $14(29.8)$ \\
\hline Severe ACS & $9(19.1)$ \\
\hline \multicolumn{2}{|l|}{ CCS, n (\%) } \\
\hline Mild CCS & $20(42.6)$ \\
\hline Moderate CCS & $14(29.8)$ \\
\hline Severe CCS & $13(27.6)$ \\
\hline
\end{tabular}

CsA, cyclosporine A; FK, tacrolimus; MMF, mycophenolic acid; Pre, prednisone; ACS, acute composite score; CCS, chronic composite score.

T2-weighted images were routinely acquired. Multi-b DWI was performed by using a respiration-triggered echoplanar sequence in the axial plane under the following parameters: TR, 2,857 ms; TE, $87.2 \mathrm{~ms}$; section thickness, $6 \mathrm{~mm}$; number of slices, 15 ; field of view (FOV) $38 \times 30.4 \mathrm{~cm}$; matrix, 256 $\times 128$; number of excitations 2 . A total of 11 diffusion gradient b values from 0 to $1,000 \mathrm{~s} / \mathrm{mm}^{2}(0,10$, $30,50,70,100,150,200,400,800,1,000)$ were applied in three diffusion directions. The total scan time for the DWI ranged from 4 to 6 minutes, depending on the respiratory rhythm of the patient.

\section{MRI image analyses}

Raw DWI images were transferred to a vendor-supplied imaging workstation (Advantage Workstation 4.6; General Electric Healthcare) and post-processed using the MADC program. Regions of interest (ROI) were drawn manually by two radiologists (YD and LP, both with more than 5 years of experience with abdominal radiology) independently and without referring to the clinical or pathologic results. Each radiologist placed a large ROI to encompass the cortex and three smaller ROIs in the medulla $\left(20-40 \mathrm{~mm}^{2}\right.$ each) in each slice for three consecutive peri-hilar slices on the $\mathrm{b}=0 \mathrm{~mm}^{2} / \mathrm{s}$ images, as we previously reported (9). A typical example of ROI placement is shown in Figure S1. The ROIs were then copied automatically to the functional maps, whose readings were later averaged to be the cortical and medullary readings for each allograft. Caution was exercised to exclude area/slices with hemorrhages, cysts, or inhomogeneous intensities when delineating ROIs.

\section{Mono-exponential and bi-exponential models of DWI}

DWI parameters were calculated on a pixel-by-pixel basis. The total apparent diffusion coefficient $\left(\mathrm{ADC}_{\mathrm{T}}\right)$ was obtained by fitting all $11 \mathrm{~b}$ values using a mono-exponential model according to the following equation: $\mathrm{S}_{\mathrm{b}} / \mathrm{S}_{0}=\exp \left(-\mathrm{b} \times \mathrm{ADC}_{\mathrm{T}}\right)$, where $S_{b}$ represents the signal intensity at a given $b$ value, and $\mathrm{S}_{0}$ is the signal intensity for $\mathrm{b}=0 \mathrm{~mm}^{2} / \mathrm{s}$. Perfusion fraction (fp), true diffusion coefficient (D), and perfusion-related pseudodiffusion coefficient $\left(\mathrm{D}^{*}\right)$ were calculated by using the biexponential intravoxel incoherent motion (IVIM) analysis: $\mathrm{S}_{\mathrm{b}} / \mathrm{S}_{0}=(1-\mathrm{fp}) \exp (-\mathrm{bD})+$ fp.exp $\left[-\mathrm{b}\left(\mathrm{D}^{*}+\mathrm{D}\right)\right]$. Consistent with earlier studies (10-12), a constrained segmented fitting algorithm was employed. Briefly, initial $D$ value was estimated using a reduced set of b values $>200 \mathrm{~s} / \mathrm{mm}^{2}$, and subsequently, D was kept constant when fitting for the determination of the fp and the pseudo-perfusion $\mathrm{D}^{*}$.

\section{Histologic analysis}

A renal allograft biopsy was performed within 1 week after MRI examinations. Slides stained for hematoxylin and eosin, periodic acid Schiff, and Masson's trichrome stains were routinely prepared for pathologic evaluation. Masson's trichrome-stained slides were digitized and quantified for tubulointerstitial fibrosis by a semi-automatic 
Table 2 Correlations between DWI parameters and histopathologic changes

\begin{tabular}{|c|c|c|c|c|c|c|}
\hline Pathologic parameters & Cortical $\mathrm{ADC}_{\mathrm{T}}$ & Medullary $\mathrm{ADC}_{\mathrm{T}}$ & Cortical D & Medullary D & Cortical fp & Medullary fp \\
\hline $\operatorname{ccs}(\rho)$ & $-0.76^{\star \star \star}$ & $-0.77^{\star \star \star}$ & $-0.63^{\star \star \star}$ & $-0.53^{\star \star \star}$ & $-0.71^{\star \star \star}$ & $-0.47^{\star \star}$ \\
\hline Interstitial fibrosis $(r)$ & $-0.79^{\star \star \star}$ & $-0.74^{\star \star \star}$ & $-0.62^{\star \star \star}$ & $-0.56^{\star \star \star}$ & $-0.59^{\star \star \star}$ & $-0.50^{\star \star \star}$ \\
\hline
\end{tabular}

${ }^{* \star}, \mathrm{P}<0.01 ;{ }^{* \star *}, \mathrm{P}<0.001$. DWI, diffusion-weighted imaging; $A D C_{T}$, total apparent diffusion coefficient; $\mathrm{D}$, true diffusion; fp, perfusion fraction; ACS, acute composite score; CCS, chronic composite score.

calculation of the percentages of areas with blue color with the colocalization algorithm to calculate the burden of interstitial fibrosis (version 9; Aperio Technologies, Inc.) in a digital pathology platform (Aperio Scanscope XT Turbo Scanner, Leica, Wetzlar, Germany) as previously reported (13). The Banff 2015 classification (14) is an international consensus regarding the diagnosis and scoring of allograft pathologies; the calculated tubulointerstitial fibrosis were categorized as mild $(<25 \%)$, moderate $(25-$ $50 \%)$ or severe $(\geq 50 \%)$.

Kidney allograft acute and chronic pathologic changes were retrieved from the pathology report that routinely reported Banff descriptor scores. The acute changes of interstitial inflammation, peritubular capillaritis, glomerulitis, and tubulitis were each scored from 0 to 3 . According to the transplantation literature, a score of 0 indicated the absence of this lesion while a score of 3 denoted the most severe changes (15). The score of each acute pathologic lesion was summed up to reach an acute composite score (ACS) that reflected the overall severity of acute changes. Similarly, a chronic composite score (CCS) was generated for better characterization of chronic pathologic changes, which included interstitial fibrosis, tubular atrophy, vascular intimal thickening, and chronic glomerulopathy. The scoring of each chronic lesion was like that described for acute pathologic lesions. A detailed description of the scoring system for acute and chronic pathologic lesions is described in Table S1. ACS and CCS were arbitrarily divided into three categories, with $0-4,5-8$, and 9-12 standing for mild, moderate, and severe changes, respectively.

\section{Statistical analysis}

The inter-rater observer agreement was assessed by calculating the intraclass correlation coefficient (ICC). The correlations between DWI parameters and tubulointerstitial fibrosis, ACS, and CCS were evaluated by the Pearson's or
Spearman's correlation analysis, as appropriate. Receiveroperating characteristic (ROC) curves were used to evaluate the diagnostic performances of both mono-exponential and bi-exponential parameters for distinguishing mild from moderate and severe ACS/CCS/tubulointerstitial fibrosis as well as mild and moderate from severe ACS/ CCS/tubulointerstitial fibrosis. The areas under the curve (AUCs) for the significant parameters were compared, as reported previously (16). We also calculated the sensitivity and specificity for parallel or serial use of $\mathrm{D}$ and $\mathrm{fp}$ at each optimal cutoff point. A $\mathrm{P}$ value of $<0.05$ was considered statistically significant in two-sided tests.

\section{Results}

\section{Measurement reproducibility}

The ICCs for cortical and medullary $\mathrm{ADC}_{\mathrm{T}}$ were 0.903 and 0.894 respectively, the ICCs of cortical D and fp were 0.837 and 0.874 respectively, and the ICCs of medullary D and fp were 0.825 and 0.883 respectively. The DWI parameters from both raters thus averaged and used for subsequent analysis.

\section{Correlations between DWI parameters and pathologic lesions}

Mono-exponential $\mathrm{ADC}_{\mathrm{T}}$ and bi-exponential $\mathrm{D} \& \mathrm{fp}$ in both the cortex and medulla were all inversely correlated with ACS, CCS, and interstitial fibrosis (Table 2). The correlation coefficient was the largest between ACS and cortical fp $(\rho=0.68)$, CCS and medullary $\operatorname{ADC}_{T}(\rho=0.77)$, and interstitial fibrosis and cortical $\mathrm{ADC}_{\mathrm{T}}(\mathrm{r}=0.79)$.

\section{Comparison of mono-exponential and bi-exponential models for assessing acute pathologic changes}

Allografts with moderate and severe ACS had significantly reduced $\mathrm{ADC}_{\mathrm{T}}$, compared with those with only mild ACS. Figure 1 illustrates typical images of DWI functional 

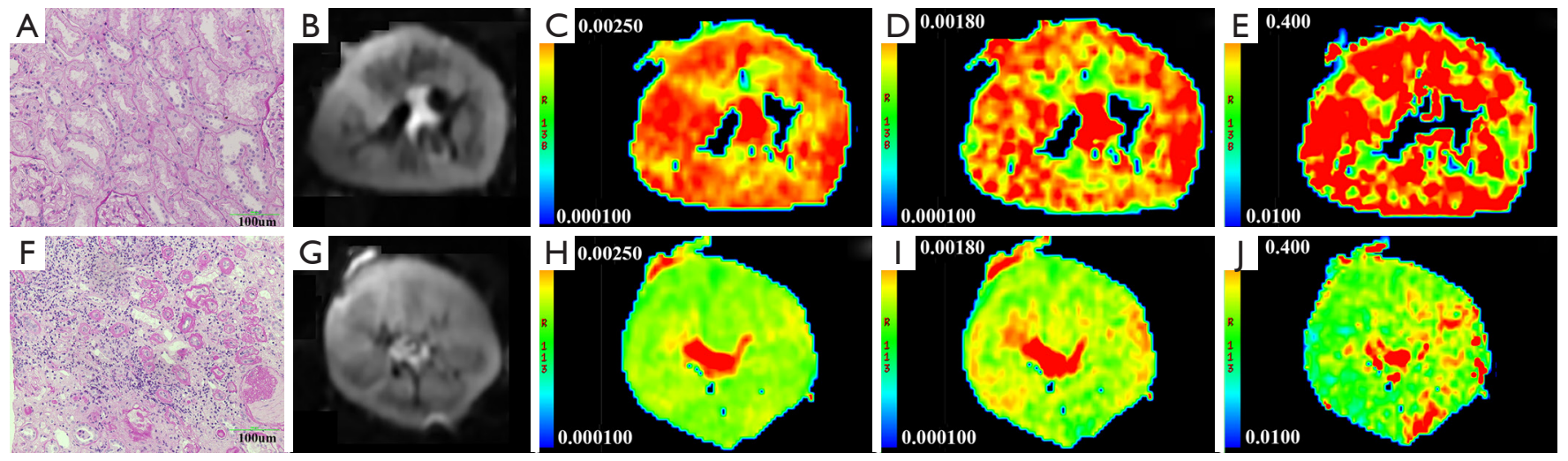

Figure 1 Pathologic slides and functional DWI maps in an allograft with no acute pathologic changes (A,B,C,D,E) and one with severe acute pathologic changes (F,G,H,I,J). (A) Preserved renal architecture with no inflammation (PAS stain, scale bar $100 \mu \mathrm{m}$ ); (B) b=0 image for the allograft shown in (A); (C,D,E) ADCT, D, and fp maps respectively for the allograft with pathology shown in (A); (F) a biopsy showing distorted renal architecture with avid inflammation (PAS stain, scale bar $100 \mu \mathrm{m})$; $(\mathrm{G}) \mathrm{b}=0$ image for the allograft shown in (F); $(\mathrm{H}, \mathrm{I}, \mathrm{J}) \mathrm{ADCT}$, D, and fp maps respectively for the allograft with pathology shown in (F); the values in $(\mathrm{H}, \mathrm{I}, \mathrm{J})$ correspondingly decreased as compared with $(\mathrm{C}, \mathrm{D}, \mathrm{E})$. DWI, diffusion-weighted imaging; ADCT, total apparent diffusion coefficient; D, true diffusion; fp, perfusion fraction.

Table 3 ROC curve analysis using DWI parameters for the discrimination of different categories of ACS

\begin{tabular}{|c|c|c|c|c|c|}
\hline Parameters & Cutoff & AUC (95\% Cl) & Sensitivity (95\% Cl) (\%) & Specificity (95\% Cl) (\%) & $Z$ static, $P$ value \\
\hline \multicolumn{6}{|l|}{ Mild vs. moderate and severe ACS } \\
\hline Cortical ADC $\mathrm{T}_{\mathrm{T}}\left(\times 10^{-3} \mathrm{~mm}^{2} / \mathrm{s}\right)$ & 2.13 & $0.76(0.61-0.87)$ & $45.8(25.6-67.2)$ & $100(85.2-100)$ & $3.4,<0.001$ \\
\hline Medullary $\mathrm{ADC}_{\mathrm{T}}\left(\times 10^{-3} \mathrm{~mm}^{2} / \mathrm{s}\right)$ & 1.85 & $0.74(0.59-0.86)$ & $58.3(36.6-77.9)$ & $82.6(61.2-95.0)$ & $3.2,0.001$ \\
\hline Cortical D $\left(\times 10^{-3} \mathrm{~mm}^{2} / \mathrm{s}\right)$ & 1.45 & $0.69(0.54-0.81)$ & $83.3(62.6-95.3)$ & $56.5(34.5-76.8)$ & $2.3,0.02$ \\
\hline Cortical fp & 0.276 & $0.89(0.77-0.96)$ & $87.5(67.6-97.3)$ & $82.6(61.2-95.0)$ & $8.4,<0.001$ \\
\hline Medullary fp & 0.252 & $0.73(0.58-0.85)$ & $87.5(67.6-97.3)$ & $52.2(30.6-73.2)$ & $3.2,0.002$ \\
\hline \multicolumn{6}{|l|}{ Severe vs. mild and moderate ACS } \\
\hline Cortical $\mathrm{ADC}_{\mathrm{T}}\left(\times 10^{-3} \mathrm{~mm}^{2} / \mathrm{s}\right)$ & 1.89 & $0.67(0.52-0.80)$ & $77.8(40.0-97.2)$ & $63.2(46.0-78.2)$ & $1.9,0.06$ \\
\hline Cortical fp & 0.276 & $0.80(0.65-0.90)$ & $100(66.4-100)$ & $65.8(48.6-80.4)$ & $4.4,<0.001$ \\
\hline Medullary fp & 0.252 & $0.66(0.51-0.80)$ & 66.7 (29.9-92.5) & $76.3(59.8-88.6)$ & $1.6,0.11$ \\
\hline
\end{tabular}

ROC, receiver-operating characteristic; DWI, diffusion-weighted imaging; ACS, acute composite score; AUC, area under the curve; Cl, confidence interval; $A D C_{T}$, total apparent diffusion coefficient; $D$, true diffusion; fp, perfusion fraction.

analysis in an allograft with mild ACS and one with severe ACS. As summarized in Table 3, ROC curve analysis indicated that cortical $\mathrm{fp}$ and $\mathrm{ADC}_{\mathrm{T}}$ distinguished mild from moderate and severe ACS with the largest AUC of 0.89
[95\% confidence interval (CI), 0.77-0.96] and the highest specificity of $100 \%$ (95\% CI, $85.2-100 \%)$. Noticeably, only cortical fp could differentiate severe ACS from mild and moderate ACS $(\mathrm{P}<0.001)$ with an AUC of 0.80 (95\% CI, 
Table 4 ROC curve analysis using DWI parameters for the discrimination of different categories of interstitial fibrosis

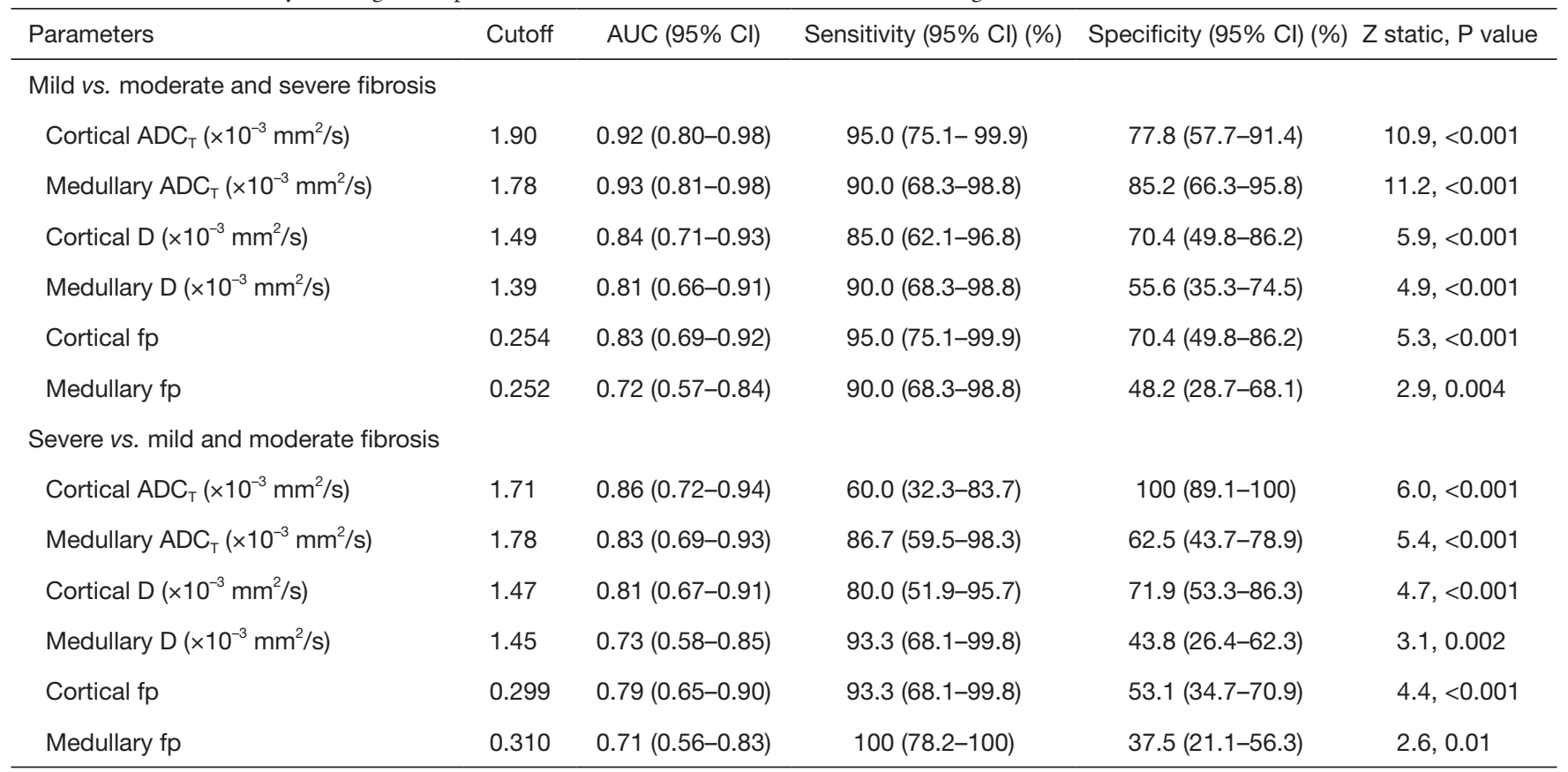

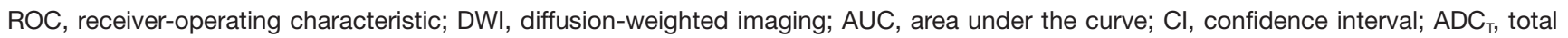
apparent diffusion coefficient; $D$, true diffusion; fp, perfusion fraction.

$0.65-0.90)$ and a sensitivity of $100 \%$ (95\% CI, 66.4-100\%). Therefore, cortical fp was superior to the $\mathrm{ADC}_{\mathrm{T}}$ for finding both mild and severe acute pathologic changes

\section{Comparison of mono-exponential and bi-exponential models for characterizing interstitial fibrosis}

When looking at the ROC curve analysis in distinguishing mild fibrosis from moderate and severe fibrosis (Table 4), AUCs for cortical and medullary $\mathrm{ADC}_{\mathrm{T}}$ were significantly different compared to medullary $\mathrm{D}(0.92$ vs. $0.81, \mathrm{P}=0.04$; 0.93 vs. $0.81, \mathrm{P}=0.03$ respectively) and $\mathrm{fp}(0.92$ vs. 0.72 , $\mathrm{P}=0.008 ; 0.93$ vs. $0.72, \mathrm{P}=0.006$ respectively), but similar to cortical D $(0.84,95 \% \mathrm{CI}, 0.71-0.93)$ and fp $(0.83$, 0.69-0.92). Furthermore, cortical $\mathrm{ADC}_{\mathrm{T}}$ had the largest AUC of 0.86 (95\% CI, 0.72-0.94) and a specificity of $100 \%$ (89.1-100.0\%) for identifying severe interstitial fibrosis. However, the AUCs by $\mathrm{ADC}_{\mathrm{T}}, \mathrm{D}$, and fp in both the cortex and medulla were not statistically significant.

\section{Comparison of mono-exponential and bi-exponential models for differentiating chronic pathologic changes}

Since interstitial fibrosis is just a part of chronic change, we then further evaluated the ability of DWI parameters to assess the CCS. Table 5 shows that both cortical $\mathrm{ADC}_{\mathrm{T}}$, medullary $\mathrm{ADC}_{\mathrm{T}}$, cortical $\mathrm{D}$, and fp distinguished mild CCS from moderate to severe CCS with good AUCs (AUC from 0.81 to 0.90 ). For identifying mild CCS, the AUC of medullary $\mathrm{ADC}_{\mathrm{T}}(0.90,95 \% \mathrm{CI}, 0.78-0.97)$ was similar to that of cortical D $(0.81,95 \% \mathrm{CI}, 0.67-0.91)$ and fp (0.86, $95 \%$ CI, 0.73-0.94), but statistically larger than that of medullary $\mathrm{D}(\mathrm{P}=0.005)$ and $\mathrm{fp}(\mathrm{P}=0.01)$. The AUCs for differentiating between severe and mild and moderate CCS were statistically insignificant among $\mathrm{ADC}_{\mathrm{T}}, \mathrm{D}$, and $\mathrm{fp}$ in the cortex and medulla $(\mathrm{P} \geq 0.15)$.

\section{Combining $D$ and fp for assessing renal allograft pathologic changes}

As shown in Table 6, a maximal sensitivity of 100\% (95\% CI, $85.8-100 \%$ ) was obtained with the parallel use of cortical D and cortical/medullary fp for distinguishing mild from moderate and severe ACS. Strikingly, the joint use of $\mathrm{D}$ and $\mathrm{fp}$ in either the cortex or the medulla could achieve a sensitivity of $100 \%$ for identifying either mild or severe interstitial fibrosis. In the meantime, the serial use of cortical $\mathrm{D}$ and cortical fp could result in the largest specificity for 
Table 5 ROC curve analysis using DWI parameters for the discrimination of different categories of CCS

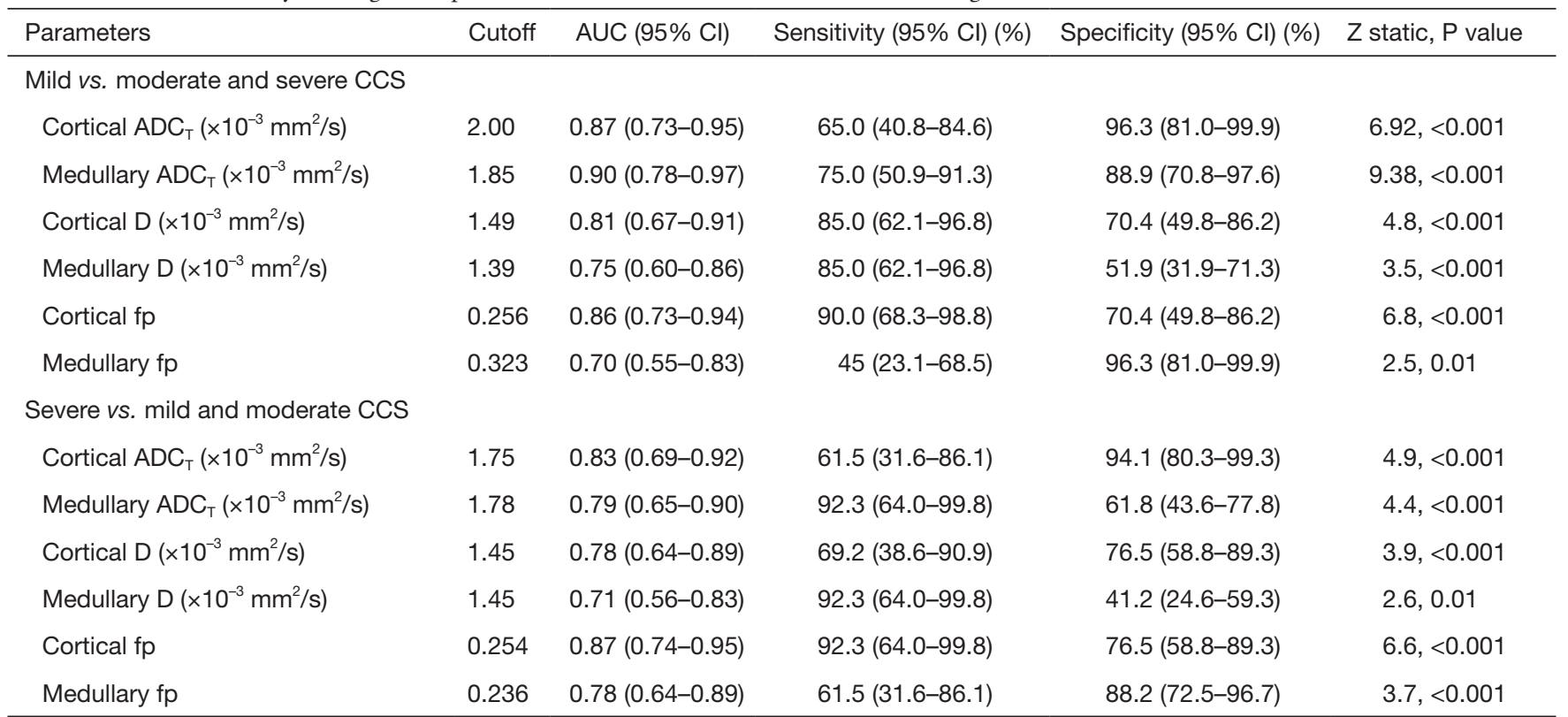

$\mathrm{ROC}$, receiver-operating characteristic; DWI, diffusion-weighted imaging; CCS, chronic composite score; AUC, area under the curve; Cl, confidence interval; $A D C_{T}$, total apparent diffusion coefficient; $D$, true diffusion; fp, perfusion fraction.

Table 6 Combining D and fp for assessing renal allograft pathologic changes

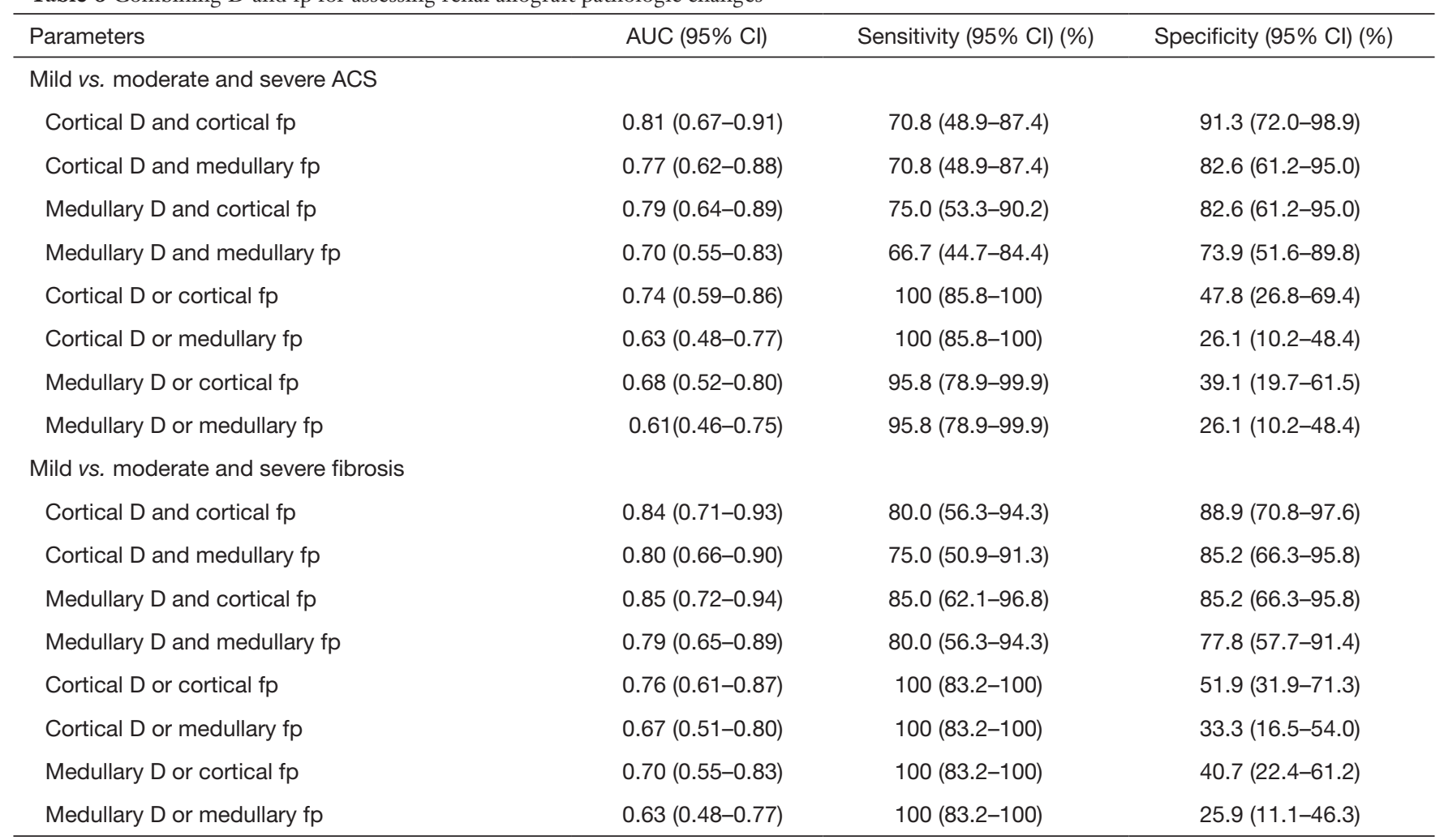

Table 6 (continued) 
Table 6 (continued)

\begin{tabular}{|c|c|c|c|}
\hline Parameters & AUC $(95 \% \mathrm{Cl})$ & Sensitivity (95\% Cl) (\%) & Specificity $(95 \% \mathrm{Cl})(\%)$ \\
\hline \multicolumn{4}{|c|}{ Severe vs. mild and moderate fibrosis } \\
\hline Cortical D and cortical fp & $0.79(0.65-0.89)$ & $73.3(44.9-92.2)$ & $84.4(67.2-94.7)$ \\
\hline Medullary D and cortical $\mathrm{fp}$ & $0.79(0.65-0.89)$ & 86.7 (59.5-98.3) & 71.9 (53.3-86.3) \\
\hline Medullary D and medullary $\mathrm{fp}$ & $0.78(0.63-0.88)$ & $93.3(68.1-99.8)$ & $62.5(43.7-78.9)$ \\
\hline Cortical D or medullary $f p$ & $0.67(0.52-0.80)$ & $100(78.2-100)$ & $34.4(18.6-53.2)$ \\
\hline Medullary D or cortical fp & $0.63(0.47-0.76)$ & $100(78.2-100)$ & $25.0(11.5-43.4)$ \\
\hline Medullary D or medullary $f p$ & $0.59(0.44-0.74)$ & $100(78.2-100)$ & $18.8(7.2-36.4)$ \\
\hline \multicolumn{4}{|l|}{ Mild vs. moderate and severe CCS } \\
\hline Medullary D and cortical fp & $0.85(0.72-0.94)$ & $85.0(62.1-96.8)$ & $85.2(66.3-95.8)$ \\
\hline Medullary D and medullary $\mathrm{fp}$ & $0.70(0.55-0.83)$ & $40.0(19.1-63.9)$ & $100(87.2-100)$ \\
\hline Cortical D or cortical fp & $0.73(0.59-0.85)$ & $95.0(75.1-99.9)$ & 51.9 (31.9-71.3) \\
\hline Cortical D or medullary $f p$ & $0.80(0.65-0.90)$ & $85.0(62.1-96.8)$ & $74.1(53.7-88.9)$ \\
\hline Medullary D or cortical fp & $0.66(0.51-0.79)$ & $95.0(75.1-99.9)$ & $37.0(19.4-57.6)$ \\
\hline Medullary D or medullary $f p$ & $0.69(0.54-0.82)$ & $90.0(68.3-98.8)$ & $48.2(28.7-68.1)$ \\
\hline \multicolumn{4}{|l|}{ Severe vs. mild and moderate CCS } \\
\hline Cortical D and cortical fp & $0.78(0.63-0.89)$ & $61.5(31.6-86.1)$ & $94.1(80.3-99.3)$ \\
\hline Medullary D or cortical fp & $0.68(0.52-0.81)$ & $100(75.3-100)$ & $35.3(19.7-53.5)$ \\
\hline Medullary D or medullary $\mathrm{fp}$ & $0.62(0.47-0.76)$ & $92.3(64.0-99.8)$ & $32.4(17.4-50.5)$ \\
\hline
\end{tabular}

$\mathrm{D}$, true diffusion; fp, perfusion fraction; AUC, area under the curve; $\mathrm{Cl}$, confidence interval; ACS, acute composite score; CCS, chronic composite score.

identifying both mild [88.9\% (95\% CI, 70.8-97.6\%)] and severe [84.4\% (95\% CI, 67.2-94.7\%)] interstitial fibrosis. Furthermore, parallel use of cortical D and cortical fp could increase the sensitivity for distinguishing mild from moderate and severe CCS to $95.0 \%$ (95\% CI, 75.1-99.9\%), whereas the serial use of medullary D and medullary $\mathrm{fp}$ could increase specificity to $100 \%$ (95\% CI, $87.2-100 \%$ ). However, for differentiating severe from mild and moderate CCS, a maximal specificity of $100 \%$ (95\% CI, $89.7-100 \%$ ) was attained by serial use of cortical D and medullary fp.

\section{Discussion}

The noninvasive assessment of renal allograft acute and chronic pathologic lesions are helpful for tailoring management and predicting prognosis. In this study, we found that cortical fp calculated by the bi-exponential model was strongly correlated with acute pathologic lesions and was superior to $\mathrm{ADC}_{\mathrm{T}}$ for identifying severe acute pathologic changes. By contrast, $\mathrm{ADC}_{\mathrm{T}}$ was equal to or better than single D or fp for characterizing interstitial fibrosis and 
chronic pathologic changes. Nonetheless, combined use of $\mathrm{D}$ and fp could increase the sensitivity and specificity in discriminating allograft pathologic changes severity.

Acute pathologic lesions are histologically characterized by inflammatory cell infiltration, forming typical features of interstitial inflammation, glomerulitis, tubulitis, and peritubular capillaritis. Clinically, these types of changes can be ameliorated or even completely dissipated with the treatment of glucocorticoids. However, the evaluation of acute pathologic changes received little attention in the literature in relation to renal DWI. Inflammatory cell infiltration may partially account for the decreased $\mathrm{ADC}_{\mathrm{T}}$, which is particularly sensitive to water diffusion restriction caused by increased cellular density. In line with this, Notohamiprodjo et al. (17) reported a significant negative correlation between $\mathrm{ADC}_{\mathrm{T}}$ and interstitial inflammation $(\mathrm{r}=-0.73)$ in rat models of kidney transplant rejection.

Although both cortical and medullary $\mathrm{ADC}_{\mathrm{T}}$ correlated with ACS and could identify early mild ACS with moderate AUC, they could not be used to distinguish severe from mild and moderate ACS. By contrast, cortical fp demonstrated a strong correlation with ACS and can be used to identify both mild and severe ACS with good AUC. According to the IVIM imaging theory proposed by Le Bihan et al. (18), fp correlated with the blood microcirculation in the capillary network. Nevertheless, studies have shown that fp in the kidney may also be related to the tubular flow (19). It may be explained by a decreased fp being related to reduced transplant capillary perfusion with an increasing ACS, as it has been reported that kidney blood microcirculation, as assessed by ultrasound, diminished in a peritubular capillaritis-dependent manner in kidney allografts (20).

Interstitial fibrosis is an irreversible lesion that has received considerable attention in the literature in regards to the native kidneys. At least a dozen studies have explored the utility of DWI for assessing interstitial fibrosis either in animal models or human subjects (4-8,13,21-27). However, the diagnostic performances of mono-exponential and biexponential models, to the best of our knowledge, have been scarcely compared. In the present study, we found that the AUCs from cortical and medullary $\mathrm{ADC}_{\mathrm{T}}$ demonstrated no significant differences with those from cortical $\mathrm{D}$ and fp, suggesting that cortical IVIM-DWI parameters and $\mathrm{ADC}_{\mathrm{T}}$ are complementary for assessing renal allograft tubulointerstitial fibrosis. These results are consistent with a prior study by Wang et al. (13) who found that both cortical $\mathrm{fp}$ and $\mathrm{ADC}_{\mathrm{T}}$ distinguished mild from moderate and severe fibrosis (0.81 vs. 0.87, $\mathrm{P}>0.05)$ and severe from mild and moderate fibrosis $(0.86$ vs. $0.88, \mathrm{P}>0.05)$ with similar AUCs. Nevertheless, combining fp and D could yield a sensitivity of $100 \%$ for identifying severe interstitial fibrosis, which was consistent with the study by Wáng et al., demonstrating that fp may be the most sensitive parameter for assessing liver fibrosis (28).

In addition to interstitial fibrosis, pathologic changes of tubular atrophy, chronic glomerulopathy, and vascular intimal thickening are also irreversible and should be routinely assessed and reported. Earlier studies have concluded that ADC correlated with chronic pathologic lesions (29), which was congruent with the good-toexcellent diagnostic performances calculated from the ROC curve analysis in the present study. Additionally, we found that $\mathrm{ADC}_{\mathrm{T}}$ was equal to or better than some IVIM-DWI parameters for evaluating chronic pathologic changes. These results emphasize that $\mathrm{ADC}_{\mathrm{T}}$ is still a robust parameter for evaluating chronic pathologic burden, but cortical D and fp could also provide useful information.

Of further interest, we showed that the combined use of $\mathrm{D}$ and fp could significantly increase the sensitivity or specificity for discriminating allograft pathologic changes severity. This result is consistent with the finding by $\mathrm{Ni}$ et al. indicating that the combined use of cortical D and medullary fp could increase the specificity in identifying allografts that need clinical intervention (30). Given that IVIM-DWI parameters D and fp denote disparate pathophysiologic processes, it is not surprising that their combined use would characterize tissue microstructure changes more accurately.

Undoubtedly, the results of this study would be influenced by the number and strength of the gradient $b$ values, for which there is no consensus to date. For instance, it is known that $\mathrm{D}$ is determined by higher $\mathrm{b}$ values $\left(>100 \mathrm{~s} / \mathrm{mm}^{2}\right)$, while $\mathrm{D}^{*}$ is significantly influenced by lower $\mathrm{b}$ values. The number of $\mathrm{b}$ values used in the literature ranges from 1 to 16 (31), with strength generally no more than $1,500 \mathrm{~s} / \mathrm{mm}^{2}(32)$.

The results of the present study are subject to several limitations. First, the ROI in this study encompassed the entire renal parenchyma, while the biopsy sampled was only from a small region. Second, the patient population was small. Third, ROIs were drawn manually, which is time-consuming and may introduce potential biases. There are other studies that have used automatic segmentation and convolutional neural networks $(33,34)$ which can increase the measurement reproducibility and reduce 
labor, respectively. Finally, the IVIM parameter $\mathrm{D}^{*}$ was not assessed in this study, since it has been reported that $D^{*}$ suffers from a notoriously low reproducibility that significantly impairs its clinical utility $(35,36)$.

In conclusion, we showed that cortical fp was superior to the $\mathrm{ADC}_{\mathrm{T}}$ for identifying both mild and severe acute pathologic changes. Nevertheless, $\mathrm{ADC}_{\mathrm{T}}$ was equal to or better than single $\mathrm{D}$ or fp for evaluating chronic pathologic changes. Thus, both mono-exponential and bi-exponential analysis of DWI images are complementary in evaluating kidney allograft pathologic changes, and the combined use of $\mathrm{D}$ and $\mathrm{fp}$ can increase the sensitivity and specificity for discriminating allograft pathologic changes severity.

\section{Acknowledgments}

Funding: This study was funded by the Applied Basic Research of Changzhou City (No. CJ20190091) with grants to MF.

\section{Footnote}

Conflicts of Interest: All authors have completed the ICMJE uniform disclosure form (available at http://dx.doi. org/10.21037/qims-19-985a). MF reports grants from Applied Basic Research of Changzhou City during the conduct of the study. The other authors have no conflicts of interest to declare.

Ethical Statement: This study was approved by the Institutional Review Board of the Third Affiliated Hospital of Soochow University. Written informed consent was obtained from all patients.

Open Access Statement: This is an Open Access article distributed in accordance with the Creative Commons Attribution-NonCommercial-NoDerivs 4.0 International License (CC BY-NC-ND 4.0), which permits the noncommercial replication and distribution of the article with the strict proviso that no changes or edits are made and the original work is properly cited (including links to both the formal publication through the relevant DOI and the license). See: https://creativecommons.org/licenses/by-nc-nd/4.0/.

\section{References}

1. Coemans M, Süsal C, Döhler B, Anglicheau D, Giral M, Bestard O, Legendre C, Emonds MP, Kuypers D,
Molenberghs G, Verbeke G, Naesens M. Analyses of the short- and long-term graft survival after kidney transplantation in Europe between 1986 and 2015. Kidney Int 2018;94:964-73.

2. Galichon P, Xu-Dubois YC, Finianos S, Hertig A, Rondeau E. Clinical and histological predictors of longterm kidney graft survival. Nephrol Dial Transplant 2013;28:1362-70.

3. Bane O, Wagner M, Zhang JL, Dyvorne HA, Orton M, Rusinek H, Taouli B. Assessment of renal function using intravoxel incoherent motion diffusion-weighted imaging and dynamic contrast-enhanced MRI. J Magn Reson Imaging 2016;44:317-26.

4. Hueper K, Khalifa AA, Bräsen JH, Vo Chieu VD, Gutberlet M, Wintterle S, Lehner F, Richter N, Peperhove M, Tewes S, Weber K, Haller H, Wacker F, Gwinner W, Gueler F, Hartung D. Diffusion-weighted imaging and diffusion tensor imaging detect delayed graft function and correlate with allograft fibrosis in patients early after kidney transplantation. J Magn Reson Imaging 2016;44:112-21.

5. Xu X, Palmer SL, Lin X, Li W, Chen K, Yan F, Li X. Diffusion-weighted imaging and pathology of chronic kidney disease: initial study. Abdom Radiol (NY) 2018;43:1749-55.

6. Mao W, Zhou J, Zeng M, Ding Y, Qu L, Chen C, Ding X, Wang Y, Fu C. Chronic kidney disease: Pathological and functional evaluation with intravoxel incoherent motion diffusion-weighted imaging. J Magn Reson Imaging 2018;47:1251-9.

7. Zhao J, Wang ZJ, Liu M, Zhu J, Zhang X, Zhang T, Li $\mathrm{S}, \mathrm{Li}$ Y. Assessment of renal fibrosis in chronic kidney disease using diffusion-weighted MRI. Clin Radiol 2014;69:1117-22.

8. Cai XR, Yu J, Zhou QC, Du B, Feng YZ, Liu XL. Use of intravoxel incoherent motion MRI to assess renal fibrosis in a rat model of unilateral ureteral obstruction. J Magn Reson Imaging 2016;44:698-706.

9. Fan M, Ni X, Li Y, Chen J, Cheng D, Shi D, He X, Wen $\mathrm{J}$. Assessment of transplant renal artery stenosis with diffusion-weighted imaging: a preliminary study. Magn Reson Imaging 2019;60:157-63.

10. Li T, Che-Nordin N, Wáng YXJ, Rong PF, Qiu SW, Zhang SW, Zhang P, Jiang YF, Chevallier O, Zhao F, Xiao $\mathrm{XY}$, Wang $\mathrm{W}$. Intravoxel incoherent motion derived liver perfusion/diffusion readouts can be reliable biomarker for the detection of viral hepatitis B induced liver fibrosis. Quant Imaging Med Surg 2019;9:371-85. 
11. Wáng YXJ, Wang X, Wu P, Wang Y, Chen W, Chen H, Li $\mathrm{J}$. Topics on quantitative liver magnetic resonance imaging. Quant Imaging Med Surg 2019;9:1840-90.

12. Ding Y, Zeng M, Rao S, Chen C, Fu C, Zhou J. Comparison of biexponential and monoexponential model of diffusion-weighted imaging for distinguishing between common renal cell carcinoma and fat poor angiomyolipoma. Korean J Radiol 2016;17:853-63.

13. Wang W, Yu Y, Wen J, Zhang M, Chen J, Cheng D, Zhang L, Liu Z. Combination of functional magnetic resonance imaging and histopathologic analysis to evaluate interstitial fibrosis in kidney allografts. Clin J Am Soc Nephrol 2019; 14:1372-80.

14. Loupy A, Haas M, Solez K, Racusen L, Glotz D, Seron D, Nankivell BJ, Colvin RB, Afrouzian M, Akalin E, Alachkar N, Bagnasco S, Becker JU, Cornell L, Drachenberg C, Dragun D, de Kort H, Gibson IW, Kraus ES, Lefaucheur C, Legendre C, Liapis H, Muthukumar T, Nickeleit V, Orandi B, Park W, Rabant M, Randhawa P, Reed EF, Roufosse C, Seshan SV, Sis B, Singh HK, Schinstock C, Tambur A, Zeevi A, Mengel M. The Banff 2015 kidney meeting report: current challenges in rejection classification and prospects for adopting molecular pathology. Am J Transplant 2017;17:28-41.

15. Li X, Chen J, Cheng D, Wang W, Xie K, Zhang M, Xu F, Wen J, Tang Z. Histopathologic features that predict transplant glomerulopathy progression in a Chinese cohort. Am J Nephrol 2019;49:425-34.

16. DeLong ER, DeLong DM, Clarke-Pearson DL. Comparing the areas under two or more correlated receiver operating characteristic curves: a nonparametric approach. Biometrics 1988;44:837-45.

17. Notohamiprodjo M, Kalnins A, Andrassy M, Kolb M, Ehle B, Mueller S, Thomas MN, Werner J, Guba M, Nikolaou K, Andrassy J. Multiparametric functional MRI: a tool to uncover subtle changes following allogeneic renal transplantation. PLoS One 2016;11:e0165532.

18. Le Bihan D, Breton E, Lallemand D, Aubin ML, Vignaud J, Laval-Jeantet M. Separation of diffusion and perfusion in intravoxel incoherent motion MR imaging. Radiology 1988;168:497-505.

19. Ebrahimi B, Rihal N, Woollard JR, Krier JD, Eirin A, Lerman LO. Assessment of renal artery stenosis using intravoxel incoherent motion diffusion-weighted magnetic resonance imaging analysis. Invest Radiol 2014;49:640-6.

20. Scholbach T, Wang HK, Yang AH, Loong CC, Wu TH. Correlation of histopathologic and dynamic tissue perfusion measurement findings in transplanted kidneys.
BMC Nephrol 2013;14:143.

21. Inoue T, Kozawa E, Okada H, Inukai K, Watanabe S, Kikuta T, Watanabe Y, Takenaka T, Katayama S, Tanaka J, Suzuki H. Noninvasive evaluation of kidney hypoxia and fibrosis using magnetic resonance imaging. J Am Soc Nephrol 2011;22:1429-34.

22. Hueper K, Rong S, Gutberlet M, Hartung D, Mengel M, Lu X, Haller H, Wacker F, Meier M, Gueler F. T2 relaxation time and apparent diffusion coefficient for noninvasive assessment of renal pathology after acute kidney injury in mice: comparison with histopathology. Invest Radiol 2013;48:834-42.

23. Friedli I, Crowe LA, Berchtold L, Moll S, Hadaya K, de Perrot T, Vesin C, Martin PY, de Seigneux S, Vallée JP.

New magnetic resonance imaging index for renal fibrosis assessment: a comparison between diffusion-weighted imaging and T1 mapping with histological validation. Sci Rep 2016;6:30088.

24. Friedli I, Crowe LA, de Perrot T, Berchtold L, Martin PY, de Seigneux S, Vallée JP. Comparison of readout-segmented and conventional single-shot for echo-planar diffusionweighted imaging in the assessment of kidney interstitial fibrosis. J Magn Reson Imaging 2017;46:1631-40.

25. Prasad PV, Li LP, Thacker JM, Li W, Hack B, Kohn O, Sprague SM. Cortical perfusion and tubular function as evaluated by magnetic resonance imaging correlates with annual loss in renal function in moderate chronic kidney disease. Am J Nephrol 2019;49:114-24.

26. Prasad PV, Li W, Raj DS, Carr J, Carr M, Thacker J, Li LP, Wang C, Sprague SM, Ix JH, Chonchol M, Block G, Cheung AK, Raphael K, Gassman J, Wolf M, Fried LF, Isakova T. Multicenter study evaluating intrarenal oxygenation and fibrosis using magnetic resonance imaging in individuals with advanced CKD. Kidney Int Rep 2018;3:1467-72.

27. Mao W, Zhou J, Zeng M, Ding Y, Qu L, Chen C, Ding X, Wang Y, Fu C, Gu F. Intravoxel incoherent motion diffusion-weighted imaging for the assessment of renal fibrosis of chronic kidney disease: a preliminary study. Magn Reson Imaging 2018;47:118-24.

28. Wáng YXJ, Deng M, Li YT, Huang H, Leung JCS, Chen $W$, Lu PX. A combined use of intravoxel incoherent motion MRI parameters can differentiate early-stage hepatitis-b fibrotic livers from healthy livers. SLAS Technol 2018;23:259-68.

29. Li Q, Li J, Zhang L, Chen Y, Zhang M, Yan F. Diffusionweighted imaging in assessing renal pathology of chronic kidney disease: a preliminary clinical study. Eur J Radiol 
2014;83:756-62.

30. Ni X, Wang W, Li X, Li Y, Chen J, Shi D, Wen J. Utility of diffusion-weighted imaging for guiding clinical management of patients with kidney transplant: a prospective study. J Magn Reson Imaging 2020. [Epub ahead of print]. doi: 10.1002/jmri.27071.

31. Caroli A, Schneider M, Friedli I, Ljimani A, De Seigneux S, Boor P, Gullapudi L, Kazmi I, Mendichovszky IA, Notohamiprodjo M, Selby NM, Thoeny HC, Grenier N, Vallée JP. Diffusion-weighted magnetic resonance imaging to assess diffuse renal pathology: a systematic review and statement paper. Nephrol Dial Transplant 2018;33:1i29-40.

32. Zhang S, Lin Y, Ge X, Liu G, Zhang J, Xu S, Wu G, Chen S, Xu J, Suo S. Multiparameter diffusion-weighted imaging for characterizing pathological patterns in lupus nephritis patients: a preliminary study. J Magn Reson Imaging 2019;50:1075-84.

33. Kim Y, Ge Y, Tao C, Zhu J, Chapman AB, Torres VE, Yu AS, Mrug M, Bennett WM, Flessner MF, Landsittel DP, Bae KT, Consortium for Radiologic Imaging Studies

Cite this article as: Fan M, Xing Z, Du Y, Pan L, Sun Y, He X. Quantitative assessment of renal allograft pathologic changes: comparisons of mono-exponential and bi-exponential models using diffusion-weighted imaging. Quant Imaging Med Surg 2020;10(6):1286-1297. doi: 10.21037/qims-19-985a of Polycystic Kidney Disease (CRISP). Automated segmentation of kidneys from MR images in patients with autosomal dominant polycystic kidney disease. Clin J Am Soc Nephrol 2016;11:576-84.

34. Sharma K, Rupprecht C, Caroli A, Aparicio MC, Remuzzi A, Baust M, Navab N. Automatic segmentation of kidneys using deep learning for total kidney volume quantification in autosomal dominant polycystic kidney disease. Sci Rep 2017;7:2049.

35. Pan J, Zhang H, Man F, Shen Y, Wang Y, Zhong Y, Ma L, Wang H, Ye H. Measurement and scan reproducibility of parameters of intravoxel incoherent motion in renal tumor and normal renal parenchyma: a preliminary research at 3.0 T MR. Abdom Radiol (NY) 2018;43:1739-48.

36. Andreou A, Koh DM, Collins DJ, Blackledge M, Wallace T, Leach MO, Orton MR. Measurement reproducibility of perfusion fraction and pseudodiffusion coefficient derived by intravoxel incoherent motion diffusion-weighted MR imaging in normal liver and metastases. Eur Radiol 2013;23:428-34. 


\section{Supplementary}

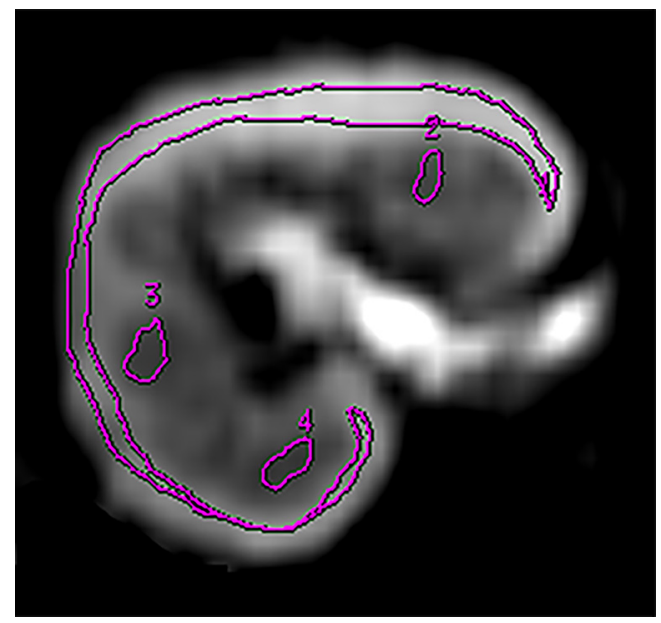

Figure S1 An example of a ROI placement in the kidney allograft. A large ROI and three smaller ROIs were manually placed in the cortex and medulla, respectively. ROI, region of interest. 
Table S1 Scoring of acute and chronic pathologic changes according to the Banff 2015 criteria

\begin{tabular}{|c|c|}
\hline Score & Pathologic changes \\
\hline \multicolumn{2}{|c|}{ Interstitial inflammation } \\
\hline 0 & No inflammation or in less than $10 \%$ of unscarred cortical parenchyma \\
\hline 1 & Inflammation in $10 \%$ to $25 \%$ of unscarred cortical parenchyma \\
\hline 2 & Inflammation in $26 \%$ to $50 \%$ of unscarred cortical parenchyma \\
\hline 3 & Inflammation in more than $50 \%$ of unscarred cortical parenchyma \\
\hline \multicolumn{2}{|c|}{ Peritubular capillaritis } \\
\hline 0 & At least one leukocyte in $<10 \%$ of cortical PTC, and/or maximum number of leukocytes $<3$ \\
\hline 1 & $\begin{array}{l}\text { At least one leukocyte cell in }>10 \% \text { of cortical PTCs with } 3-4 \text { leukocyte in most } \\
\text { severely involved PTC }\end{array}$ \\
\hline 2 & At least one leukocyte in $>10 \%$ of cortical PTC with 5-10 leukocyte in most severely involved PTC \\
\hline 3 & at least one leukocyte in $>10 \%$ of cortical PTC with $>10$ leukocyte in most severely involved PTC \\
\hline \multicolumn{2}{|c|}{ Glomerulitis } \\
\hline 0 & No glomerulitis \\
\hline 1 & Glomerulitis in less than $25 \%$ of glomeruli \\
\hline 2 & Segmental or global glomerulitis in $25 \%$ to $75 \%$ of glomeruli \\
\hline 3 & Glomerulitis in more than $75 \%$ of glomeruli \\
\hline \multicolumn{2}{|c|}{ Tubulitis } \\
\hline 0 & No mononuclear leukocytes in tubules \\
\hline 1 & Foci with 1-4 leukocytes/tubular cross section (or 10 tubular cells) \\
\hline 2 & Foci with 5-10 leukocytes/tubular cross section (or 10 tubular cells) \\
\hline 3 & Foci with $>10$ leukocytes/tubular cross section \\
\hline \multicolumn{2}{|c|}{ Interstitial fibrosis } \\
\hline 0 & Interstitial fibrosis in up to $5 \%$ of cortical area \\
\hline 1 & Interstitial fibrosis in $6 \%$ to $25 \%$ of cortical area (mild interstitial fibrosis) \\
\hline 2 & Interstitial fibrosis in $26 \%$ to $50 \%$ of cortical area (moderate interstitial fibrosis) \\
\hline 3 & Interstitial fibrosis in $>50 \%$ of cortical area (severe interstitial fibrosis) \\
\hline \multicolumn{2}{|c|}{ Tubular atrophy } \\
\hline 0 & No tubular atrophy \\
\hline 1 & Tubular atrophy involving up to $25 \%$ of the area of cortical tubules (mild tubular atrophy) \\
\hline 2 & Tubular atrophy involving $26 \%$ to $50 \%$ of the area of cortical tubules (moderate tubular atrophy) \\
\hline 3 & Tubular atrophy involving in $>50 \%$ of the area of cortical tubules (severe tubular atrophy) \\
\hline \multicolumn{2}{|c|}{ Vascular intimal thickening } \\
\hline 0 & No chronic vascular changes \\
\hline 1 & Vascular narrowing of up to $25 \%$ luminal area by fibrointimal thickening \\
\hline 2 & Vascular narrowing of $26 \%$ to $50 \%$ luminal area by fibrointimal thickening \\
\hline 3 & Vascular narrowing of more than $50 \%$ luminal area by fibrointimal thickening \\
\hline \multicolumn{2}{|c|}{ Chronic glomerulopathy } \\
\hline 0 & No GBM double contours by light microscopy or electron microscopy \\
\hline 1 & $\begin{array}{l}\text { Double contours of the GBM in } 1-25 \% \text { of capillary loops in the most affected non-sclerotic } \\
\text { glomerulus by light microscopy }\end{array}$ \\
\hline 2 & Double contours affecting $26 \%$ to $50 \%$ of peripheral capillary loops in the most affected glomerulus \\
\hline 3 & $\begin{array}{l}\text { Double contours affecting more than } 50 \% \text { of peripheral capillary loops in the most affected } \\
\text { glomerulus }\end{array}$ \\
\hline
\end{tabular}

PTC, peritubular capillaries; GBM, glomerular basement membrane. 